\title{
ULTRAMETRIC SETS IN EUCLIDEAN POINT SPACES*
}

\author{
MIROSLAV FIEDLER ${ }^{\dagger}$
}

Dedicated to Hans Schneider on the occasion of his seventieth birthday.

Abstract. Finite sets $S$ of points in a Euclidean space the mutual distances of which satisfy the ultrametric inequality $\rho(A, B) \leq \max \{\rho(A, C), \rho(C, B)\}$ for all points in $S$ are investigated and geometrically characterized. Inspired by results on ultrametric matrices and previous results on simplices, connections with so-called centered metric trees as well as with strongly isosceles right simplices are found.

AMS subject classifications. 51K05, 05C12.

Key words. Ultrametric matrices, isosceles, simplex, tree.

1. Introduction. In the theory of metric spaces (cf. [1]), a metric $\rho$ is called ultrametric if

$$
\rho(A, B) \leq \max (\rho(A, C), \rho(C, B))
$$

holds for all points $A, B, C$, of the space.

Let us observe already at this point that if $A, B$ and $C$ are mutually distinct points, then they are vertices of an isosceles triangle in which the length of the base does not exceed the lengths of the legs. This is the reason why ultrametric spaces are also called isosceles spaces.

It is well known [5, Theorem 1] that every finite ultrametric space consisting of $n+1$ (distinct) points can be isometrically embedded into a point Euclidean $n$ dimensional space but not into such an $(n \Leftrightarrow 1)$-dimensional space. We reprove this theorem and, in addition, find a complete geometric characterization of such sets.

Suppose thus that we are given a finite set $S$ of points in a point Euclidean $n$ dimensional space. We say that this set is ultrametric if the mutual distances satisfy (1) for all triples of points in $S$.

In the sequel, we use a result on strictly ultrametric matrices [6], [8]; these are real symmetric and (entrywise) nonnegative matrices $\left(a_{i k}\right)$ satisfying

$$
\begin{aligned}
a_{i k} & \geq \min \left(a_{i j}, a_{j k}\right) \text { for all } i, j, k, \\
a_{i i} & >\max _{k \neq i} a_{i k} \text { for all } i .
\end{aligned}
$$

THEOREM 1.1. [6], [8]. Every strictly ultrametric matrix $A$ is nonsingular and its inverse $B=\left(b_{i k}\right)$ is a diagonally dominant symmetric $M$-matrix, i.e. $b_{i k} \leq 0$ for all $i, k, i \neq k$, and $b_{i i}>\sum_{k \neq i}\left|b_{i k}\right|$ for all $i$.

Let us recall now some known facts from the Euclidean distance geometry and qualitative simplex geometry.

Fact 1 . Let $A_{1}, \ldots, A_{m}$ be points in a Euclidean space $\mathcal{E}$. Then these points are linearly independent if and only if

$$
\operatorname{det}\left(\begin{array}{cc}
0 & e^{T} \\
e & M
\end{array}\right) \neq 0
$$

*Received by the editors on 14 January 1998. Accepted on 11 March 1998. Handling editor: Daniel Hershkowitz.

${ }^{\dagger}$ Institute of Computer Science Czech Academy of Sciences, Pod vodárenskou věží 2, 18207 Praha 8, The Czech Republic (fiedler@math.cas.cz). This research was supported by grant GACR $201 / 95 / 1484$. 
where $e$ is a column vector of $m$ ones, $e^{T}$ its transpose and $M$ is the $m$-by- $m$ Menger matrix the $i, k$-th entry of which is the square of the distance $\rho\left(A_{i}, A_{k}\right)$.

Let, for $n \geq 2, A_{1}, \ldots, A_{n+1}$, be linearly independent points in a point Euclidean $n$-space $\mathcal{E}_{n}$, thus forming the set of vertices of an $n$-simplex $\Sigma$. By Fact 1 , the $(n+2)$ by- $(n+2)$ matrix $\widetilde{M}$ in the left-hand side of (2) is nonsingular. Consider the matrix $\widetilde{Q}=\left(q_{r s}\right), r, s=0,1, \ldots, n+1$ such that

$$
\widetilde{Q}=\Leftrightarrow 2 \widetilde{M}^{-1} .
$$

This matrix has the following geometric meaning [4].

Fact 2. a) $q_{00}=4 r^{2}, r$ being the radius of the circumscribed sphere to $\Sigma$;

b) the numbers $\Leftrightarrow \frac{1}{2} q_{0 k}$ are the barycentric coordinates of the circumcenter of $\Sigma$;

c) the matrix $Q=\left(q_{i k}\right), i, k=1, \ldots, n+1$ is the Gram matrix (the matrix of mutual inner products) of the $n+1$ outward normals to the $(n \Leftrightarrow 1)$-dimensional faces of $\Sigma$, normalized so that their sum is the zero vector. Thus, $Q$ is positive semidefinite with rank $n$ and the vector $(1, \ldots, 1)^{T}$ is -up to a nonzero factor- the only nonzero vector $x$ for which $Q x=0$.

A certain converse also holds (as before, $e$ is the column vector of ones).

Fact 3. To every $(n+1)$-by- $(n+1)$ real symmetric positive semidefinite matrix $Q$ with rank $n$ satisfying $Q e=0$ there exists in $\mathcal{E}_{n}$, up to congruence in $\mathcal{E}_{n}$, a unique $n$-simplex $\Sigma$ for which the construction above yields this matrix $Q$.

The crucial property of the matrix $Q$ is that it determines the dihedral interior angles of the simplex $\Sigma$. Indeed, the dihedral interior angle $\phi_{i k}$ between the $(n \Leftrightarrow 1)$ dimensional faces opposite to $A_{i}$ and $A_{k}(i \neq k)$ adds with the angle of the outward normals to these faces to $\pi$ so that

$$
\cos \phi_{i k}=\Leftrightarrow \frac{q_{i k}}{\sqrt{q_{i i}} \sqrt{q_{k k}}} .
$$

For sake of completeness, we recall and provide a complete answer to the question of characterizing all possible distributions of acute, right and obtuse angles in an $n$ simplex [2]. To make the result more transparent, we transfer these distributions to the edges of $\Sigma$ : we call the edge $A_{i} A_{k}(i \neq k)$ positive if the opposite dihedral angle $\phi_{i k}$ is acute, the edge will be called negative if the opposite angle is obtuse, and the edge will be neutral if the opposite angle is right. We then refer to signs of the edges (the neutral edges will have sign zero).

THEOREM 1.2. [2] In every n-simplex, the positive edges form a connected set on the set of all vertices. This condition is also sufficient in the sense that whenever we assign signs to edges of an $n$-simplex in such a way that the positive edges form a connected set on all vertices, then there exists a position of the vertices that realizes these signs.

Proof. Denote $N=\{1, \ldots, n+1\}$. Suppose that the set of positive edges does not connect the set of vertices $\left\{A_{i}\right\}, i \in N$. This means that there exists a subset $M$ of $N, \emptyset \neq M \neq N$, such that in the previous notation, none of the angles $\phi_{i k}, i \in M$, $k \in N \backslash M$, is acute. By (4), $q_{i k} \geq 0$ whenever $i \in M, k \in N \backslash M$. Since $Q e=0$, we have $\sum_{k} q_{i k}=0$ for all $i$. Thus $\sum_{i \in M} \sum_{k} q_{i k}=0$ which can be written as

$$
\sum_{i, k \in M} q_{i k}+\sum_{i \in M} \sum_{k \in N \backslash M} q_{i k}=0 .
$$

This is impossible since the second sum is nonnegative whereas the first is positive: it is the value of the quadratic form $\sum_{i, k} q_{i k} x_{i} x_{k}$ for the vector $z=\left(z_{i}\right), z_{i}=1$ for 
$i \in M, z_{k}=0$ otherwise, and $z$ is non-zero and not a multiple of $e$.

To prove the converse, denote by $E^{+}, E^{-}$respectively, the (symmetric) subsets of $N \times N$ corresponding to positive, resp. negative edges. Suppose that $E^{+}$connects the set of vertices. The quadratic form

$$
\sum_{i, k, i<k,(i, k) \in E^{+}}\left(x_{i} \Leftrightarrow x_{k}\right)^{2}
$$

is clearly positive semidefinite and -because of connectedness- equal to zero only if the vector $\left(x_{i}\right)$ is a multiple of $e$. The matrix $Q^{+}$of this form is thus positive semidefinite with rank $n$ and $Q^{+} e=0$. Let similarly $Q^{-}$be defined for the analogous quadratic form corresponding to summing for $(i, k) \in E^{-}$.

By basic properties of positive semidefinite matrices, all principal minors of order at most $n$ of $Q^{+}$are positive. It follows that for some sufficiently small positive $\varepsilon$ the same will be true for the matrix $Q=Q^{+}+\varepsilon Q^{-}$. Since $Q e=0$, this matrix $Q$ satisfies the assumptions of Fact 3 . The corresponding simplex will have edges of prescribed signs which completes the proof.

REMARK 1.3. Observe that we assigned to an $n$-simplex (with $n+1$ vertices) a signed (undirected) graph $G_{s}=\left(N, E^{+}, E^{-}\right)$whose set of vertices is $N$ and sets of positive, resp. negative edges are $E^{+}, E^{-}$, respectively. (Here, and in the sequel, we omit the neutral edges.) By Theorem 1.2, the positive subgraph $G_{s}^{+}=\left(N, E^{+}\right)$is connected. By elementary graph theory, the number of edges in $E^{+}$is at least $n$ and in the case that it is $n$, the graph $G_{s}^{+}$is a tree.

COROLLARY 1.4. [3], [4]. Every $n$-simplex has at least $n$ acute interior dihedral angles. There exist $n$-simplices which have exactly $n$ interior dihedral angles acute and all the remaining (i.e., $\left(\begin{array}{l}n \\ 2\end{array}\right)$ ) right.

Simplices mentioned in the second part of Corollary 1.4 were called right simplices in [3]. The edges opposite to acute dihedral angles (i.e., positive edges) were called legs. Thus the legs form a tree, geometrically a set of segments not containing any closed polygon and connecting the set of vertices of the simplex. Analogously to the two-dimensional case, the face determined by all end- vertices of the set of legs will be called the hypotenuse of the right simplex. In [3], we proved the following theorem.

THEOREM 1.5. [3] Any two legs of a right $n$-simplex are perpendicular. In other words, the set of legs can be completed to the set of edges of a rectangular $n$-dimensional parallelepiped. Conversely, any connected subset of edges of a rectangular n-dimensional parallelepiped no two of which are parallel forms the set of legs of a right $n$-simplex.

REMARK 1.6. It follows from the Pythagorean theorem that the Menger matrix $M$ defined above is identical with the distance matrix of the graph $G_{s}$ (which is a tree) if we assign to each edge the square of the length of the corresponding leg.

COROLLARY 1.7. Let a simplex $H$ with $n \geq 3$ vertices be the hypotenuse of a right simplex the corresponding graph of which contains vertices of degree two. Then $H$ is also the hypotenuse of a right simplex $\Sigma$ the corresponding graph of which has no vertices of degree two. The number of vertices in $\Sigma$ does not exceed $2 n \Leftrightarrow 2$.

Proof. The possibility of restriction follows from Remark 1.6. Let now $m$ be the number of vertices of the tree which does not contain vertices of degree two. The number $e$ of edges of this tree satisfies $e=m \Leftrightarrow 1$ as well as $2 e \geq n+3(m \Leftrightarrow n)$. This implies $m \leq 2 n \Leftrightarrow 2$.

REMARK 1.8. Observe that the center of the parallelepiped is at the same time the circumcenter of the right simplex. 
An easy consequence of this fact is the following result.

THEOREM 1.9. [3] The barycentric coordinates of the circumcenter of a right $n$-simplex are $c_{i}$, where for $i=1, \ldots, n+1, c_{i}=1 \Leftrightarrow \frac{1}{2} d_{i}, d_{i}$ denoting the number of legs incident with the vertex $A_{i}$. The square of the diameter of the circumscribed hypersphere is equal to the sum of squares of the lengths of all legs.

Confronting the formula (3) with the results in Theorem 1.5, Fact 2 and the expression for the Menger matrix from Remark 1.6 one sees easily the following fact.

Fact 4. In the case of a right $n$-simplex, the matrix $Q=\left(q_{i k}\right)$ has the form

$$
\begin{aligned}
q_{i k} & =\left(\rho\left(A_{i}, A_{k}\right)\right)^{-2} \text { if } A_{i} A_{k} \text { is a leg; } \\
q_{i k} & =0 \text { if } i \neq k \text { and } A_{i} A_{k} \text { is not a leg; } \\
q_{i i} & =\Leftrightarrow \sum_{k \neq i} q_{i k} \quad \text { for all } i .
\end{aligned}
$$

We conclude this section with an important fact about simplices without obtuse interior dihedral angles. In this case, the set of negative edges $E^{-}$is void and the graph $G_{s}$ has positive edges only. Such simplices were called in [7] hyperacute angled; we call them hyperacute.

THEOREM 1.10. [3] Let $\Sigma$ be a hyperacute $n$-simplex with the set of vertices $\left\{A_{i}\right\}, i \in N$. Let $M$ be a subset of $N$. Then the face of $\Sigma$ determined by the vertices $\left\{A_{i}\right\}, i \in M$, is again a hyperacute simplex. Its graph $G_{1}$ is uniquely determined by the graph $G$ of $\Sigma$ as follows. The set of its vertices is $M$; two distinct vertices $p$ and $q$ are connected if and only if there exists in $G$ a path from $p$ to $q$ such that all vertices of this path, except $p$ and $q$, belong to the set $N \backslash M$.

2. Results. We first introduce a special kind of metric trees, i.e. trees every edge of which has a positive length. In such a tree, the distance of two vertices is simply the sum of lengths of edges in the unique path between these vertices. We call a metric tree centered if it has a vertex $C$ which has the same distance from all end-vertices of the tree.

It is evident that in a centered metric tree $T$ the vertex $C$ is unique; we call it the center of $T$ and its distance from end-vertices is the radius of $T$. In addition, the diameter of $T$, i.e., the maximum distance between two vertices, is twice the radius of $T$. For our purpose, we agree to consider as such centered metric tree also a graph with a single vertex which is at the same time an end-vertex and the center, the radius being zero.

The following lemma is easily proved.

LEMMA 2.1. Let $V$ be a vertex of a centered tree $T$, let $T_{V}$ be the induced subgraph of $T$ on the set of those vertices $W$ of $T$ for which the path from $C$ to $W$ contains $V$. Then $T_{V}$ is again a centered metric tree and its center is $V$.

We can now formulate a theorem the essence of which was proved in [8].

THEOREM 2.2. (Basic theorem on ultrametrics.) Let $D=\left(\rho_{i k}\right)$ be a real symmetric $n-b y-n$ matrix. Then the following are equivalent.

(a) The numbers $\rho_{i k}$ are mutual distances of some ultrametric set of $n$ distinct points.

(b) There exists a centered metric tree with at most $2 n \Leftrightarrow 1$ vertices and exactly $n$ end-vertices, such that $\rho_{i k}$ are mutual distances between these end-vertices.

(c) There exists a centered metric tree with at most $2 n \Leftrightarrow 1$ vertices and exactly $n$ end-vertices, with center $C$ and radius $r$ such that for any pair $i, k$ of end-vertices, $r \Leftrightarrow \frac{1}{2} \rho_{i k}$ is the distance of $C$ to the path between $i$ and $k$ (for $i=k$, the path reduces to the point i). 
Proof. (a) $\Rightarrow$ (b). We use induction in $n$. For $n=1$ and $n=2$, the implication is true. Suppose that $n>2$ and that the implication is true for smaller positive integers. Let $A_{1}, \ldots, A_{n}$, be points of the ultrametric set $U_{0}$. Let $\rho_{0}=\max _{i, k} \rho\left(A_{i}, A_{k}\right)$. Define a relation $R_{0}$ in the set $N_{0}=\{1, \ldots, n\}$ by

$$
i R_{0} k \Longleftrightarrow \rho\left(A_{i}, A_{k}\right)<\rho_{0} .
$$

The relation $R_{0}$ is reflexive, symmetric and, by (1), transitive. Let $M_{1} \cup M_{2} \cup \ldots \cup M_{s}$ be the decomposition of $N_{0}$ into classes of equivalence with respect to $R_{0}$. The sets $U_{k}=\left\{A_{j}, j \in M_{k}\right\}, k=1, \ldots, s$, are again ultrametric and since $s>1$, each of them contains less than $n$ points. By the induction hypothesis, there exist metric trees $T_{k}$, $k=1, \ldots, s$, with centers $C_{k}$ and radii $r_{k}$ such that the mutual distances between their $\left|M_{k}\right|$ end-vertices are the corresponding $\rho_{p q}$ 's and $r_{k}=\frac{1}{2} \max _{p, q \in M_{k}} \rho\left(A_{p}, A_{q}\right)$; observe that all the $r_{k}$ 's are smaller than $\frac{1}{2} \rho_{0}$. We now construct a centered tree $T_{0}$ from the trees $T_{k}$ by joining each vertex $C_{k}$ with a new vertex $C_{0}$ by an edge of length $r_{0} \Leftrightarrow r_{k}$. The tree $T_{0}$ is indeed centered with center $C_{0}$ since the distance of an endvertex in, say, $M_{i}$, from $C_{i}$ is $r_{i}$, from $C_{i}$ to $C_{0}$ is $\frac{1}{2} \rho_{0} \Leftrightarrow r_{i}$, so that its distance from $C_{0}$ is $r_{0}=\frac{1}{2} \max _{p, q \in N_{0}} \rho\left(A_{p}, A_{q}\right)$. It is also immediate that the distance between two end-vertices in different $M_{k}$ 's is $\rho_{0}$. Finally, the number of vertices in $T_{0}$ is $2 \sum_{k}\left|M_{k}\right| \Leftrightarrow s+1$ which does not exceed $2 n \Leftrightarrow 1$.

(b) $\Rightarrow(\mathrm{c})$. Take the same tree $T_{0}$ in (c) as we have in (b). We again use induction. Let $i, j$ be end-vertices. If $i=j$, the assertion in (c) is correct. Let thus $i \neq j$. If the path from $i$ to $j$ in $T_{0}$ contains $C_{0}$, then $\rho_{i k}=2 r_{0}$ and the assertion is again correct. Let now the path from $i$ to $j$ not contain $C_{0}$. Then both $i$ and $j$ belong to some $M_{k}$ in the previous notation. By the induction hypothesis, the distance of $C_{k}$ to the path is $r_{k} \Leftrightarrow \frac{1}{2} \rho_{i j}$. Since the distance between $C_{0}$ and $C_{k}$ is $r_{0} \Leftrightarrow r_{k}$, the result is as asserted. (c) $\Rightarrow$ (a). Let a centered metric tree be given. It suffices to show that for any triple $p, q, r$, of distinct end-vertices, at least two of the distances $d_{p q}, d_{p r}, d_{q r}$, of the paths from the center $C$ are equal and the third is at least that long. Let $\widetilde{C}$ be the last vertex in common to all three paths from $C$ to the end-vertices; of the first vertices $C_{p}, C_{q}, C_{r}$, of these paths to $p, q, r$, next to $\widetilde{C}$ at least one, say $C_{p}$, is different from each of the other two. Then both $d_{p q}, d_{p r}$, are equal to the distance from $C$ to $\widetilde{C}$ and $d_{q r}$ is not smaller.

We proceed now to the case of Euclidean spaces.

THEOREM 2.3. Every ultrametric set of distinct points in a Euclidean space is formed by linearly independent points.

Proof. Let $M$ be the Menger matrix of the given points forming an ultrametric set, and let $\widetilde{M}$ be the matrix

$$
\left(\begin{array}{ll}
0 & e^{T} \\
e & M
\end{array}\right)
$$

By Fact 1 , we have to prove that $\operatorname{det} \widetilde{M} \neq 0$. To this end, it suffices to show an equivalent statement that

$$
\operatorname{det}\left(\begin{array}{cc}
0 & e^{T} \\
e & \sigma e e^{T} \Leftrightarrow M
\end{array}\right) \neq 0 \text { for some } \sigma \text {. }
$$

Choose $\sigma$ greater than the square of the maximum distance between the given points. The matrix $\sigma e e^{T} \Leftrightarrow M$ will then be strictly ultrametric. Its inverse $B=\left(b_{i k}\right)$ is by 
Fact 2 a diagonally dominant $M$-matrix and thus satisfies

$$
\sum_{k} b_{i k}>0 \text { for all } i
$$

We have then, I being the identity matrix,

$$
\begin{aligned}
\operatorname{det}\left(\begin{array}{cc}
0 & e^{T} \\
e & \sigma e e^{T} \Leftrightarrow M
\end{array}\right) & =\operatorname{det}\left(\begin{array}{cc}
0 & e^{T} \\
e & B^{-1}
\end{array}\right) \\
& =\operatorname{det} B^{-1} \operatorname{det}\left(\begin{array}{cc}
0 & e^{T} \\
B e & I
\end{array}\right) \\
& =\Leftrightarrow \operatorname{det} B^{-1}\left(e^{T} B e\right) \\
& =\Leftrightarrow \operatorname{det} B^{-1} \sum_{i, k} b_{i k} .
\end{aligned}
$$

Since this sum is positive, the proof is complete.

In the sequel, we thus consider ultrametric simplices only, i.e., simplices the vertices of which form an ultrametric set. Having in mind the relationship between trees and right simplices mentioned in Theorem 1.5 and Remark 1.6, we introduce a notion which corresponds to that of the centered metric tree.

A right simplex $\Sigma$ is called right strongly isosceles if one of its vertices $C$, called quasicenter, has the same distance from all vertices of the hypotenuse $H$ of $\Sigma$.

REMARK 2.4. It is easily seen that an equivalent property of such a right simplex is that for some vertex, the quasicenter, the line connecting it with the circumcenter of the hypotenuse is perpendicular to the hypotenuse. Observe that, due to Remark 1.8 , the center of the parallelepiped generated by the simplex is also a point of this line.

We can now formulate our main result.

THEOREM 2.5. The vertices of the hypotenuse of any strongly isosceles right simplex form an ultrametric set of (at least two) points. Conversely, every ultrametric set of $n \geq 2$ distinct points in a Euclidean space forms the set of vertices of the hypotenuse of a strongly isosceles right simplex of dimension at most $2 n \Leftrightarrow 2$.

Proof. Let $\Sigma$ be a strongly isosceles right simplex the hypotenuse $H$ of which has $n$ vertices. Clearly, $n \geq 2$. The set of legs of $\Sigma$ forms a tree $T$. If we assign to each leg as a new (graphic) length the square of its geometric length, then $T$ can be considered as a metric tree. By the Pythagorean theorem, the square of the geometric distance between any two vertices of the hypotenuse is equal to the graphic distance in $T$. Since $\Sigma$ is isosceles, $T$ is centered. By Theorem 2.2, the distances between the end-vertices of $T$ satisfy (1). The same is thus true for the geometric distances between the $n$ vertices of the hypotenuse. By Corollary $1.7, H$ is even the hypotenuse of some right simplex with at most $2 n \Leftrightarrow 1$ vertices, since the quasicenter might be incident with only two legs. This simplex is easily seen to be again strongly isosceles. The converse follows also from Theorem 2.2. $\square$

THEOREM 2.6. Every ultrametric simplex is hyperacute.

Proof. It follows from Theorems 2.5 and 1.10 .

We conclude this section by an apparently new characterization of the distance matrix of a general ultrametric set formed by $n$ distinct points.

THEOREM 2.7. Let $D=\left(d_{i k}\right)$ be an $n \times n$ matrix. Then the following are equivalent.

1. $D$ is the distance matrix of an ultrametric set of $n$ distinct points. 
2. There exist $n \Leftrightarrow 1$ positive numbers $a_{1}, \ldots, a_{n-1}$, and a permutation $P$ of the indices $1, \ldots, n$, so that $d_{k k}=0$ for $k=1, \ldots, n$, and

$$
\text { for } 1 \leq i<k \leq n, d_{P i, P k}=d_{P k, P i}=\max \left\{a_{j} ; i \leq j<k\right\}
$$

Proof. It is immediate that 2. implies 1 . To prove the converse, we use induction in $n$. The implication $1 . \Rightarrow 2$. holds for $n=1$ and $n=2$. Suppose that $D=\left(d_{i k}\right)$ is an ultrametric distance matrix corresponding to $n>2$ distinct points and that the implication holds for all such matrices of smaller order. $D$ is nonnegative symmetric and has zero entries only in the diagonal. Let $d_{p q}$ be a maximum entry in $D$; we can assume that $p<q$. Define in the set of indices $N=\{1, \ldots, n\}$ a relation $R$ by

$$
i R k \Leftrightarrow d_{i k}<d_{p q}
$$

This relation is reflexive, symmetric and, by ultrametricity of $D$, also transitive. Let $N=N_{1} \cup N_{2} \cup \ldots \cup N_{r}$ be the decomposition of $N$ into classes of equivalence with respect to $R$, the first, say, $s$ of them having more than one element, the remaining only one. Reorder $N$ so that we start with entries in $N_{1}$ and continue till $N_{r}$. If $s=0$, all off-diagonal entries of $D$ are equal to $d_{p q}$ and we can take $a_{1}=\ldots=a_{n-1}=d_{p q}$. Let thus $s \geq 1$, let $m=\left|N_{1}\right|$. In the reordered matrix $\tilde{D}$ the upper-left-corner $m \times m$ submatrix is again ultrametric corresponding to distinct points. By the induction hypothesis, there exist positive numbers $a_{1}, \ldots, a_{m-1}$, and an ordering of indices in $N_{1}$ so that (5) holds. The same holds also for $N_{2} \cup \ldots \cup N_{r}$ with some numbers $\hat{a}_{1}, \ldots, \hat{a}_{n-m-1}$. Set now $a_{m}=d_{p q}, a_{m+k}=\hat{a}_{k}, k=1, \ldots, n \Leftrightarrow m \Leftrightarrow 1$. For the appropriate ordering, (5) will hold for the whole matrix $D$. $\square$

REMARK 2.8. The ordering of points in this "interval representation" is not unique. For instance, the two points determining the interval with (one of) the smallest $a_{i}$ can be switched. On the other hand, if we have an interval representation of an ultrametric set $S$ and if $S_{1}$ is a subset of $S$, then an interval representation of $S_{1}$ can be obtained by preserving only the points corresponding to $S_{1}$, and setting the value of the interval $I$ between the two consecutive points in $S_{1}$ as the maximum of the values of the original intervals whose union is $I$.

3. Realization of Ultrametric Sets. We end the paper by showing how to realize ultrametric sets in Cartesian coordinates of the Euclidean space.

Let $D=\left(d_{i k}\right)$ be the matrix of mutual distances of $n \geq 3$ points satisfying the ultrametric inequalities. Using the partitioning algorithm from the proof of Theorem 2.2 , we construct a centered metric tree $T$ without vertices of degree two (except possibly the center) which has exactly $n$ end-vertices and such that the mutual distances are the squares $d_{i k}^{2}$. (This is possible since the squares again satisfy the ultrametric inequality.) The total number $m$ of its vertices thus satisfies $n<m \leq 2 n \Leftrightarrow 1$ by Corollary 1.7 .

Let $V_{1}, \ldots, V_{m}$ be the vertices of $T, E_{1}, \ldots, E_{m-1}$ its edges. We can assume that $V_{1}, \ldots, V_{n}$ are the end-vertices and $V_{m}$ the center of $T$. To each vertex $V_{s}$ we assign its radius $r_{s}$ and to each edge $E_{k}=\left(V_{p}, V_{q}\right)$ its length $\sigma_{k}=\left|r_{p} \Leftrightarrow r_{q}\right|$.

We now construct a set of $m$ points $W_{1}, \ldots, W_{m}$ in the Euclidean $(m \Leftrightarrow 1)$ dimensional space with the usual orthonormal basis and corresponding Cartesian coordinates as follows.

The point $W_{m}$ has all coordinates zero. For $s>1$, let $\left(V_{m}, E_{k_{1}}, V_{j_{1}}, \ldots, E_{k_{t}}, V_{j_{t}}\right)$, $j_{t}=s$, be the path from $V_{m}$ to $V_{s}$ in $T$. The coordinates $\left(a_{1 s}, \ldots, a_{m-1, s}\right)$ of $W_{s}$ are 
given by

$$
\begin{aligned}
& a_{j s}=\sqrt{\frac{1}{2} \sigma_{k_{p}}} \text { if } j=k_{p} \text { for some } p, 1 \leq p \leq t, \\
& a_{j s}=0 \text { otherwise. }
\end{aligned}
$$

Let us show that the points $W_{k}$ form the set of vertices of a right and even strongly isosceles ( $m \Leftrightarrow 1)$-simplex whose set of vertices of the hypotenuse solves our problem.

Observe that if $\left(V_{i}, V_{k}\right)$ is an edge in $T$, then the coordinate vectors of $W_{i}$ and $W_{k}$ differ in exactly one coordinate. The set of edges of $T$ thus corresponds to the set of legs of a right ( $m \Leftrightarrow 1)$-simplex with vertices $W_{1}, \ldots, W_{m}$. In addition, the square of the length of each leg is equal to the length of the corresponding edge of $T$. The property of $T$ to be centered implies that this simplex is strongly isosceles with the quasicenter $W_{m}$.

Let us show that the mutual distances of the vertices of its hypotenuse form in the appropriate ordering the matrix $D$.

Let thus $W_{i}, W_{j}$ be distinct vertices of its hypotenuse, thus corresponding to end-vertices $V_{i}, V_{j}$ of $T$. Let $V_{k}$ be the closest vertex of the path between $V_{i}$ and $V_{j}$ to $V_{m}$. We have then by the definition of $W_{k}$ and Theorem 2.2 that the square of the distance between $W_{m}$ and $W_{k}$ is $r \Leftrightarrow \frac{1}{2} d_{i j}^{2}$. Since the squares of the distances between $W_{m}$ and each $W_{i}, W_{j}$ are equal to $r$, we obtain by Pythagorean theorem that $\left|W_{i} W_{k}\right|^{2}=\left|W_{j} W_{k}\right|^{2}=\frac{1}{2} d_{i j}^{2}$ and thus $\left|W_{i} W_{j}\right|=d_{i j}$ as asserted.

\section{REFERENCES}

[1] J. Dieudonné, Fondements de l'analyse moderne, Gautier-Villars, Paris, 1968.

[2] M. Fiedler, Geometry of the simplex in $E_{n}$, Part I. Časopis Pěst. Mat. 79:297-320, 1954. In Czech.

[3] M. Fiedler, Über qualitative Winkeleigenschaften der Simplexe. Czechoslovak Math. Journal $7: 463-478,1957$.

[4] M. Fiedler, Über die qualitative Lage des Mittelpunktes der umgeschriebenen Hyperkugel im n-Simplex. Comm. Math. Univ. Carol. 2:3-51, 1961.

[5] Lemin, A. Yu., Isometric embedding of isosceles (non-Archimedian) spaces into Euclidean ones. Dokl. Akad. Nauk SSSR 285:558-562, 1985. In Russian. English Translation: Sov. Math. Dokl. 32:740-744, 1985.

[6] S. Martínez, G. Michon, and J. San Martin, Inverses of ultrametric matrices are of Stieltjes type. SIAM J. Matrix Anal. Appl. 15:98-106, 1994.

[7] D. J. H. Moore and G. E. Subak-Sharpe, Metric transformation of an $(m+1)$-terminal resistive network into a hyperacute angled simplex $P_{m}$ in Euclidean space $E_{m}$. In Proceedings of the Eleventh Midwest Symposium on Circuit Theory, Notre Dame, Indiana, May 13-14, 1968, Univ. of Notre Dame, pp. 184-192.

[8] R. Nabben and R. S. Varga, A linear algebra proof that the inverse of a strictly ultrametric matrix is a strictly diagonally dominant Stieltjes matrix. SIAM J. Matrix Anal. Appl. $15: 107-113,1994$. 